\title{
Evolution of the $\bar{K} N-\pi \Sigma$ system with $M_{\pi}^{2}$ in a box from $\mathbf{U} \chi \mathbf{P T}$
}

\section{R. Molina ${ }^{1}$ and M. Döring ${ }^{1,2}$}

${ }^{1}$ Department of Physics, The George Washington University, $72521^{\text {st }}$ St. NW, Washington, DC 20052, USA. ${ }^{2}$ Thomas Jefferson National Accelerator Facility, 12000 Jefferson Ave, Newport News, VA 23606.

E-mail: ramope71@gwu.edu

The $\Lambda(1405)$ baryon is difficult to detect in experiment, absent in many quark model calculations, and supposedly manifested through a two-pole structure. Its uncommon properties made it subject to numerous experimental and theoretical studies in recent years. Finite-volume Lattice-QCD eigenvalues for different quark masses were recently reported by the Adelaide group. We compare these eigenvalues to predictions of a model based on Unitary Chiral Perturbation Theory. The UCHPT calculation predicts the quark mass dependence remarkably well. It also explains the overlap pattern with different meson-baryon components, mainly $\pi \Sigma$ and $\bar{K} N$, at different quark masses. More accurate Lattice QCD data are required to draw definite conclusions on the nature of the $\Lambda(1405)$.

The 8th International Workshop on Chiral Dynamics, CD2015 ***

29 June 2015 - 03 July 2015

Pisa,Italy 


\section{Introduction}

The $\Lambda(1405)$ has been considered as a quasibound molecular state of the $\bar{K} N$ system for many years [1,2]. In fact, there are experimental evidences that the $\Lambda(1405)$ resonance, which has been observed in the $\pi \Sigma$ invariant mass distribution, is mostly a $\bar{K} N$ and/or $\pi \Sigma$ composite [3-7]. The reason is that the $\Lambda(1405)$ lies just $25 \mathrm{MeV}$ below the $\bar{K} N$ threshold and has a strong influence in the low energy $\bar{K} N$ data $[3,4,6,7]$. The new kaonic hydrogen measurements by SIDDHARTA [8], together with total cross section data and threshold branching ratios, are successfully described in the framework of chiral SU(3) coupled-channels dynamics with input based on the NLO mesonbaryon effective Lagrangian [9], where the experimental data are used to constraint the mesonbaryon coupled channel amplitudes, giving rise to a more precise determination of the location of the two poles. Implications of the new data for $K d$ scattering are discussed in Refs. [10,11].

Since the $\Lambda(1405)$ mass lies between the $\pi \Sigma$ and $\bar{K} N$ thresholds, a coupled-channel description is mandatory. In fact, all the unitary frameworks based on chiral Lagrangians for the study of the $S$ - wave meson baryon interaction lead to the generation of this resonance $[9,12-20]$. Within the $U \chi P T$ framework, two poles close to the $\Lambda(1405)$ resonance mass appear. This was also the case in the cloudy bag model of Ref. [21]. The coupled-channel formalism takes into account all possible pseudoscalar meson-baryon channels with $I=0 ; J=1 / 2$ (except for $\eta^{\prime} \Lambda$ whose coupling is supposed to be negligible): $\bar{K} N, \pi \Sigma, \eta \Lambda$ and $K \Xi$ [16,19,22]. For example, in Ref. [22] the two states are found in the complex plane of scattering energy at $\sqrt{s}=(1390-66 i) \mathrm{MeV}$ and $(1426-16 i) \mathrm{MeV}$. Both states lie on the same Riemann sheet, with the real parts of their pole positions above the $\pi \Sigma$ and below the $\bar{K} N$ threshold. In most approaches the lower state is wider and couples stronger to the $\pi \Sigma$ channel, while the upper state close to the $\bar{K} N$ threshold is narrower and couples stronger to the $\bar{K} \mathrm{~N}$ threshold. The position and width of the lighter state is less well determined than in case of the heavier state [20,23].

Evidence of the proposed two pole structure has been noticed through the study of different reactions $[13,18,24]$. Indeed, the two pole structure can be studied by means of different production reactions which favor one or the other pole [25, 26]. For instance, the reaction $\pi^{-} p \rightarrow K^{0} \pi \Sigma$ studied in Ref. [27] shows a different shape of the resonance, and is dominated by the $\pi \Sigma \rightarrow \pi \Sigma$ amplitude, hence, favoring the lower and wider state. Further evidence for two $\Lambda(1405)$ states is found in $[28,29]$. The composite nature of the $\Lambda(1405)$ as $\bar{K} N$ bound state has been investigated in $[30,31]$.

The finite-volume spectrum of the $\Lambda(1405)$ was predicted in Ref. [40] based on a dynamical coupled-channel model and a chiral unitary approach. The coupled-channel $\bar{K} N, \pi \Sigma$ scattering lengths in the finite volume were discussed in Ref. [41].

Recently, the spectrum of excited hyperons became accessible in ab-initio simulations of QCD on the lattice [32-38]. The quark mass dependence of a scattering level close to the $\Lambda(1405)$ position was determined in Ref. [39].

The aim of the study is to test the two-pole hypothesis of the $\Lambda(1405)$ in the light of the new lattice QCD data from Ref. [39]. For this, we study the evolution of the poles found in the $T$ matrix of the meson-baryon system with Isospin $=0$, Strangeness $=-1$ and $J=1 / 2$, using the lowest order potential from $\mathrm{U} \chi \mathrm{PT}$ in the finite volume, with $M_{\pi}^{2}$, for several sets of ground state masses, the ones used in Ref. [39], and the physical-mass set. We will study the properties of the first two 
states, pole positions, distances to the $\bar{K} N$ and $\pi \Sigma$ thresholds, and couplings to the meson-baryon components, and compare to the lattice data.

\section{The $\Lambda(1405)$ as dynamically generated resonance in the infinite volume}

In the chiral unitary approach, the $\Lambda(1405)$ resonance is dynamically generated in $s$-wave meson-baryon scattering from the set of coupled channels, $\bar{K} N, \pi \Sigma, \eta \Lambda$ and $K \Xi$. The scattering equation used to study the meson-baryon system is [13]

$$
T=(1-V G)^{-1} V
$$

where the matrix $V$ is the interaction kernel of the scattering equation, and we take the $s$-wave interaction given by the lowest order of chiral perturbation theory (the Weinberg-Tomozawa interaction),

$$
V_{i j}(W)=-C_{i j} \frac{1}{4 f_{i} f_{j}}\left(2 W-M_{i}-M_{j}\right) \sqrt{\frac{M_{i}+E_{i}}{2 M_{i}}} \sqrt{\frac{M_{j}+E_{j}}{2 M_{j}}}
$$

with the channel indices $i, j$, the baryon mass $M$, the meson decay constants $f_{i}$, the baryon energy $E$ and the center of mass energy $W$ in the meson-baryon system. The coefficients $C_{i j}$ are the couplings strengths to the meson-baryon channels, $\bar{K} N, \pi \Sigma, \eta \Lambda$ and $K \Xi$, of each reaction $P_{i} B_{i} \rightarrow P_{j} B_{j}(i, j=$ $1,4)$, determined by the lowest-order chiral Lagrangian, $C_{i j}=\left(\left(3,-\sqrt{\frac{3}{2}}, \frac{3}{\sqrt{2}}, 0\right),\left(-\sqrt{\frac{3}{2}}, 4,0, \sqrt{\frac{3}{2}}\right)\right.$, $\left.\left(\frac{3}{\sqrt{2}}, 0,0,-\frac{3}{\sqrt{2}}\right),\left(0, \sqrt{\frac{3}{2}},-\frac{3}{\sqrt{2}}, 3\right)\right)$. The diagonal matrix $G_{i}^{D R}$ is the meson baryon loop function, evaluated using dimensional regularization as [13]

$$
\begin{aligned}
& G_{i}^{D R}(W)=i \int \frac{d^{4} q}{(2 \pi)^{4}} \frac{2 M_{i}}{q^{2}-M_{i}^{2}+i \varepsilon} \frac{1}{(P-q)^{2}-m_{i}^{2}+i \varepsilon} \\
& =\frac{2 M_{i}}{16 \pi^{2}}\left\{a_{i}(\mu)+\ln \frac{M_{i}^{2}}{\mu^{2}}+\frac{m_{i}^{2}-M_{i}^{2}+W^{2}}{2 W^{2}} \ln \frac{m_{i}^{2}}{M_{i}^{2}}+\frac{q_{\mathrm{cm}}}{W}\left[\ln \left(W^{2}-\left(M_{i}^{2}-m_{i}^{2}\right)+2 q_{\mathrm{cm}} W\right)\right.\right. \\
& +\ln \left(W^{2}+\left(M_{i}^{2}-m_{i}^{2}\right)+2 q_{\mathrm{cm}} W\right)-\ln \left(-W^{2}+\left(M_{i}^{2}-m_{i}^{2}\right)+2 q_{\mathrm{cm}} W\right) \\
& \left.\left.-\ln \left(-W^{2}-\left(M_{i}^{2}-m_{i}^{2}\right)+2 q_{\mathrm{cm}} W\right)\right]\right\},
\end{aligned}
$$

where $m$ are the meson masses, $q_{\mathrm{cm}}$ is the 3-momentum of the meson or baryon in the center of mass frame and $\mu$ is the scale of dimensional regularization. The remaining finite constant terms denoted by $a_{i}(\mu)$ are determined phenomenologically by a fit in order to reproduce the threshold branching ratios of $K^{-} p$ to $\pi \Lambda$ and $\pi \Sigma$ observed by stopped $K^{-}$mesons in hydrogen [42,43], as done in Refs. [13-15]. The $a_{i}$ constants were determined in Ref. [14] using the same decay constant for all the pseudoscalar mesons involved, $f=1.123 f_{\pi}$. However, we use here different decay constants $f_{i}, f_{j}$ in Eq. (2.2) depending of which mesons are in the external legs of the pseudoscalar (P)- baryon (B) interaction, $P_{i} B_{i} \rightarrow P_{j} B_{j}$. These constants are $f_{\pi}=92.4 \mathrm{MeV}, f_{K}=112.7 \mathrm{MeV}$ and $f_{\eta}=122.4$ $\mathrm{MeV}$ for physical masses, and are obtained for unphysical masses using the SU(3) chiral unitary extrapolation of Ref. [44]. The subtraction constants used here are $a_{\bar{K} N}=-2.2, a_{\pi \Sigma}=-1.6$, $a_{\eta \Lambda}=-2.5, a_{K \Xi}=-2.9$. These values result in very similar amplitudes as in Ref. [14], for which the $\Lambda(1405)$ is supposed to be described predominatly by meson-baryon components [31]. In 


\begin{tabular}{lcccc}
\hline \hline$W_{0}$ & $\bar{K} N$ & $\pi \Sigma$ & $\eta \Lambda$ & $K \Xi$ \\
\hline $1379-71 i$ & 2.2 & 3.1 & 0.8 & 0.5 \\
$1412-20 i$ & 3.1 & 1.7 & 1.5 & 0.3 \\
$1672-18 i$ & 0.8 & 0.3 & 1.1 & 3.4 \\
\hline \hline
\end{tabular}

Table 1: Coupling constants $\left|g_{i}\right|$ to the meson-baryon channels obtained as the residua of the scattering amplitude at the pole position.

addition, these values are close to a natural value equivalent to the three-momentum cut-off of 630 $\mathrm{MeV}$ [13].

The amplitudes $T^{i j}$ obtained from Eq. (2.1) can be analytically continued to the complex plane of the scattering energy $W$. For this, the loop functions $G_{i}$ are analytically continued along the right-hand cut into the lower $W$ plane $(\operatorname{Im} W<0$ ) according to

$$
G_{i}^{I I}(W)= \begin{cases}G_{i}(W)+i \frac{2 M_{i} q_{\mathrm{cm}}}{4 \pi W}, & \text { for } \operatorname{Re} W>m_{i}+M_{i} \\ G_{i}(W), & \text { else }\end{cases}
$$

to ensure that the resonance poles closest to the physical axis are searched for. The residua $a_{-1}^{i j}$ of the poles factorize channel-wise, $a_{-1}^{i j}=g_{i} g_{j}$, defining the coupling strengths $g_{i}$ of the resonance to the meson-baryon channels. The scattering amplitude for the channels $i$ and $j$ close to the resonance can be approximated as $T_{i j} \simeq g_{i} g_{j} /\left(W-W_{0}\right)$. As in Refs. $[13,17,18]$ the amplitude in the present study exhibits two poles, at $W_{0}=1379-71 i$ and $1412-20 i \mathrm{MeV}$. Both poles are situated on the same Riemann sheet. As the size of the couplings in Table 1 shows, the lighter state couples predominantly to the $\pi \Sigma$ channel while the heavier state couples stronger to the $\bar{K} N$ channel. If the transitions between these channels are set to zero, the lighter state is still present as a resonance in the $\pi \Sigma$ channel while the heavier state becomes a bound state in the $\bar{K} N$ channel. This demonstrates that each pole can be undertood as dynamically generated from the respective channel. The pole position of the $\Lambda(1670)$ is obtained here at $W_{0}=1672-18 i \mathrm{MeV}$. It appears as a quasi-bound $K \Xi$ state as the large coupling in Table 1 indicates.

\section{Formalism in finite volume}

One can also evaluate the loop function $G$ in Eqs. (2.1), (2.3) with a cutoff [47],

$$
G(W)=\int_{0}^{q_{\max }} \frac{d^{3} q}{(2 \pi)^{3}} \frac{\omega_{1}+\omega_{2}}{2 \omega_{1} \omega_{2}} \frac{2 M_{i}}{W^{2}-\left(\omega_{1}+\omega_{2}\right)^{2}+i \varepsilon}
$$

where $\omega_{i}=\sqrt{m_{i}^{2}+|\vec{q}|^{2}}$ is the energy and $\vec{q}$ stands for the momentum of the meson in the center of mass frame. A formalism of the $\mathrm{U} \chi \mathrm{PT}$ description of the scalar meson sector in the finite volume was introduced in Ref. [45]. Here we follow the same procedure replacing the infinite volume amplitude $T$ by the amplitude $\tilde{T}$ in a cubic box of size $L$ and $G(W)$ in Eqs. (2.3) and (3.1) is replaced by the finite volume loop function denoted as $\tilde{G}$, given by the discrete sum over 
eigenstates of the box

$$
\tilde{G}(W)=\frac{2 M_{i}}{L^{3}} \sum_{\vec{q}_{l}} I(W, \vec{q})
$$

with

which is quantized as

$$
I(W, \vec{q})=\frac{\omega_{1}(\vec{q})+\omega_{2}(\vec{q})}{2 \omega_{1}(\vec{q}) \omega_{2}(\vec{q})} \frac{1}{W^{2}-\left(\omega_{1}(\vec{q})+\omega_{2}(\vec{q})\right)^{2}}
$$

$$
\vec{q}=\frac{2 \pi}{L} \vec{n}
$$

corresponding to the periodic boundary conditions. Here the vector $\vec{n}$, denotes the three-dimensional vector of all integers $\left(\mathbb{Z}^{3}\right)$. This form produces a degeneracy for the set of three integers which have the same modulus, $q^{2}=\frac{4 \pi^{2}}{L^{2}} m$ (here $q \equiv|\vec{q}|$ and $m$ stands for the natural numbers). This multiplicity of the degeneracy should be conveniently introduced in Eq. (3.2), which can be done using the theta-series of a cubic lattice [40]. The sum over the momenta is limited by $q_{\max }$, such that $m_{\max }=\frac{q_{\max } L}{2 \pi}$. As in the infinite volume, the formalism should also be made independent of $q_{\max }$ and related to $a(\mu)$, the parameter of the dimensional regularization function loop, $G^{D R}$. This is done in Ref. [46], obtaining,

$$
\tilde{G}=G^{D R}+\lim _{q_{\max } \rightarrow \infty}\left(\frac{1}{L^{3}} \sum_{q<q_{\max }} I\left(P^{0}, \vec{q}\right)-\int_{q<q_{\max }} \frac{d^{3} q}{\left(2 \pi^{3}\right)} I\left(P^{0}, \vec{q}\right)\right) \equiv G^{D R}+\lim _{q_{\max } \rightarrow \infty} \delta G,
$$

where $\delta G \equiv \tilde{G}-G$, and $G$ is defined in Eq. 3.1. $\delta G$ is finite as $q_{\max } \rightarrow \infty$. The Bethe-Salpeter equation in finite volume can be written as,

$$
\tilde{T}=(I-V \tilde{G})^{-1} V
$$

The energy levels in the box in the presence of interaction $V$ correspond to the condition

$$
\operatorname{det}(1-V \tilde{G})=0 .
$$

In a single channel, Eq. (3.7) leads to poles in the $\tilde{T}$ amplitude when $V^{-1}=\tilde{G}$. As a consequence, an infinite number of poles is predicted for a particular box size.

For one channel, the amplitude in infinite volume $T$ for the energy levels $\left(W_{j}\right)$ can be written as

$$
T=\left(\tilde{G}\left(W_{j}\right)-G\left(W_{j}\right)\right)^{-1} .
$$

The couplings to the meson-baryon channels of the bound states in the box are evaluated as the residues of $\tilde{T}$, as in the infinite volume case.

\section{Results}

The energy levels in a box are evaluated by means of Eq. (3.7), which requires the potential of Eq. (2.2) and the meson-baryon function loop in the finite volume, $\tilde{G}$, from Eq. (3.5), which needs the functions (2.3) and (3.3). The previous expressions are used for the different pseudoscalar meson masses that are used in [39] where the pion has masses from 170 to $620 \mathrm{MeV}$. Although the pseudoscalar-meson mass dependence is explicit in $\tilde{G}$, we must take into account the dependence 
of the decay constants $f_{\pi}, f_{K}$ or $f_{\eta}$ with the mass of the pseudoscalar mesons in the potential, Eq. (2.2). This dependence can be evaluated through the $S U(3)$ unitary chiral extrapolation of [44]. In [44], the meson decay constants are related to the leading order masses and the low-energy constants that multiply the tree level diagrams from the $\mathscr{O}\left(p^{4}\right)$ lagrangian in the expansion of the meson-meson scattering amplitude $t(s)=t_{2}(s)+t_{4}(s)+\ldots$. The leading order masses can be obtained by solving the equations that relate the physical masses with the leading order masses, the LECs and the pion decay constant in the chiral limit, $f_{0}$. Here, $f_{0}$ is fixed to reproduce the physical point, and we obtain $f_{0}=79.2 \mathrm{MeV}$. The decay constants for each pseudoscalar meson mass from the lattice calculation [39] that we obtain using the SU(3) chiral extrapolation are shown in Table 2 .

Results are shown in Fig. 1. The two first energy levels from the $\mathrm{U} \chi \mathrm{PT}$ calculation are shown in the left figure in Fig. 1 together with the lattice data of [39]. Lattice data correspond to a size of the box $L \simeq 3 \mathrm{fm}$ (see Table 2). For the physical point a larger size of the box, $L=4 \mathrm{fm}$, has been used. From this figure we observe that there is good agreement between the U $\chi$ PT prediction and the lattice data for masses below $400 \mathrm{MeV}$. Actually, for larger masses the chiral extrapolation breaks down, which we show just to notice the discrepancies between $U \chi P T$ and Lattice data for large pion masses. However, we extracted two energy levels in this energy region, what is naturally expected since there are two thresholds, $\pi \Sigma$ and $\bar{K} N$, while the lattice data correspond to only the second energy level. This highlights the need for more lattice data around these energies. That could be obtained by using moving frames.

In order to understand the role of the meson-baryon channels in the energy levels extracted, we evaluate the couplings, the residua of the amplitude in the finite volume, $\tilde{T} \sim g_{i} g_{j} /\left(W-W_{0}\right)$ at the pole position. They are shown in the right figure of Fig. 1. Here the left panel of bar diagrams represents the couplings of the lowest energy level in the box, and the right one is for the second energy level. In this figure the size of the couplings are depicted for pion masses in the range $170-515 \mathrm{MeV}$, sets 1 to 4 in Table 2, from top to bottom. From the left panel we observe that the lowest state couples more significantly to $\pi \Sigma$ and $\bar{K} N$ for pion masses below $400 \mathrm{MeV}$, with the strength to $\pi \Sigma$ being slightly larger. For larger masses, this trend is inverted and the $\bar{K} N$ strength becomes larger. On the other hand, the couplings of the second energy level (right panel) show a significant dominance of the $\bar{K} N$ component. Thus, from Fig. 1, is clear that the first two Lattice data points in Fig. 1 correspond to the second energy level, for which the $\bar{K} N$ component clearly dominantes, while the third Lattice data point could belong to either the first or second energy level predicted from $\mathrm{U} \chi \mathrm{PT}$. The relatively small $\pi \Sigma$ and $\bar{K} N$ strengths in the lowest level for light pion masses can be a difficulty in order to extract this level from Lattice calculations.

We can compare with the results in the infinite volume using the formalism described in Section II together with the SU(3) chiral extrapolation explained before. The results are shown in Fig. 2. Here, the pole positions, real and imaginary part, obtained for different pion masses as a function of the pion mass in the infinite volume limit are depicted. The solid lines represent the $\pi \Sigma, \bar{K} N, \eta \Lambda$ and $K \Xi$ thresholds. For masses close to the physical point, the lower state is a resonance above the $\pi \Sigma$ threshold, while in the finite volume the lowest level is below this threshold and hence a bound state. When the mass of the pion increases, the lower state becomes a cusp in the infinite volume and a bound state when the pion mass increases further. On the other hand, the higher-energy state is always below and close to the $\bar{K} N$ threshold for all pion masses considered. In Table 4, we show 


\begin{tabular}{llrrrrrrrrrr}
\hline \hline Set & $L(f m)$ & $m_{\pi}$ & $m_{K}$ & $m_{\eta}$ & $m_{N}$ & $m_{\Lambda}$ & $m_{\Sigma}$ & $m_{\Xi}$ & $f_{\pi}$ & $f_{K}$ & $f_{\eta}$ \\
\hline 1 & 2.99 & 170.29 & 495.78 & 563.97 & 962.2 & 1135.8 & 1181.5 & 1323.6 & 94.5 & 113.2 & 122.1 \\
2 & 3.04 & 282.84 & 523.26 & 581.72 & 1058.7 & 1173.4 & 1235.5 & 1332.8 & 102.5 & 116.1 & 122.3 \\
3 & 3.08 & 387.81 & 559.46 & 605.97 & 1150.1 & 1261.0 & 1292.4 & 1377.4 & 109.5 & 118.5 & 122.6 \\
4 & 3.23 & 515.56 & 609.75 & 638.07 & 1274.5 & 1333.4 & 1353.5 & 1401.8 & 116.3 & 120.6 & 122.4 \\
5 & 3.27 & 623.14 & 670.08 & 685.01 & 1420.3 & 1434.2 & 1449.8 & 1472.4 & 120.1 & 121.9 & 122.6 \\
\hline \hline
\end{tabular}

Table 2: Pseudoscalar meson decay constants obtained from the SU(3) chiral extrapolation with the masses used in [39]. Units are MeV.

the comparison between the two first pole positions and coupling constants in the infinite and finite volume. In this table, $b_{\overline{K N}}$ and $b_{\pi \Sigma}$ denotes the distances to the $\bar{K} N$ and $\pi \Sigma$ thresholds, where the negative sign means that the state is above threshold. For masses below $400 \mathrm{MeV}$, we observe that the second state in the finite volume has also a dominant $\bar{K} N$ component, and is also between both $\pi \Sigma$ and $\bar{K} N$ thresholds. This state has similar coupling strengths to the meson-baryon components than in the infinite volume. On the contrary, the lower state shows very different properties in the infinite volume limit and in the box for low pion masses, and similar ones for high pion masses. For low pion masses, the state in the infinite volume limit is a resonance while it is a bound state in the box, where the main channels are $\pi \Sigma$ and $\bar{K} N$. However, the couplings to these channels are quite small compared to what one observes in the infinite volume limit (and also to the $\bar{K} N$ coupling of the higher energy state from the lattice data). For high pion masses, the state in the infinite volume limit becomes a bound state, and then the couplings to all the channels become very similar to the ones in the box as one can see from Table 4. However, in this case the masses of the poles obtained are very far away from the Lattice data probably because the chiral extrapolation breaks down.

\section{Conclusions}

In this work we have studied the $M_{\pi}^{2}$ dependence of the energy levels in a box for the system of meson-baryon coupled channels with quantum numbers Isospin $=0$, Strangeness $=-1$ and $J=0$ using the lowest order potential from $\mathrm{U} \chi \mathrm{PT}$. This dependence has been compared to the lattice data of [39] and also extrapolated to the infinite volume. U $\chi \mathrm{PT}$ predics a two-pole structure for the $\Lambda(1405)$. In the finite volume, we find two energy levels close to the $\pi \Sigma$ and $\bar{K} N$ thresholds. The second energy level agrees very well with the lattice data of Ref. [39] for pion masses below $400 \mathrm{MeV}$, in the limits of applicability of the theory. This energy level shows large coupling to $\bar{K} N$ and has similar properties as the higher pole of the state predicted by U $\chi \mathrm{PT}$ in the infinite volume limit and related to the $\Lambda(1405)$. The lower state predicted by the U $\chi \mathrm{PT}$ is not found in the lattice calculation of Ref. [39]. In addition, the lower state shows as a bound state below the $\pi \Sigma$ threshold (i. e. as a threshold level), while there is no level in the finite volume of the first pole at the energy of the $\Lambda(1405)$, that is a resonance dominated by the $\pi \Sigma$ channel in U $\chi$ PT. For high pion masses the lower pole in the infinite volume changes to a bound state below the $\pi \Sigma$ threshold with very similar coupling stregths in the infinite and finite volume. This work stresses the difficulty of finding resonances in Lattice calculations and urges the need for more theoretical progress in this side. It also shows the difficulty to extract the ground state from Lattice QCD in this sector, where definitely more lattice data are needed in order to understand the structure of the 

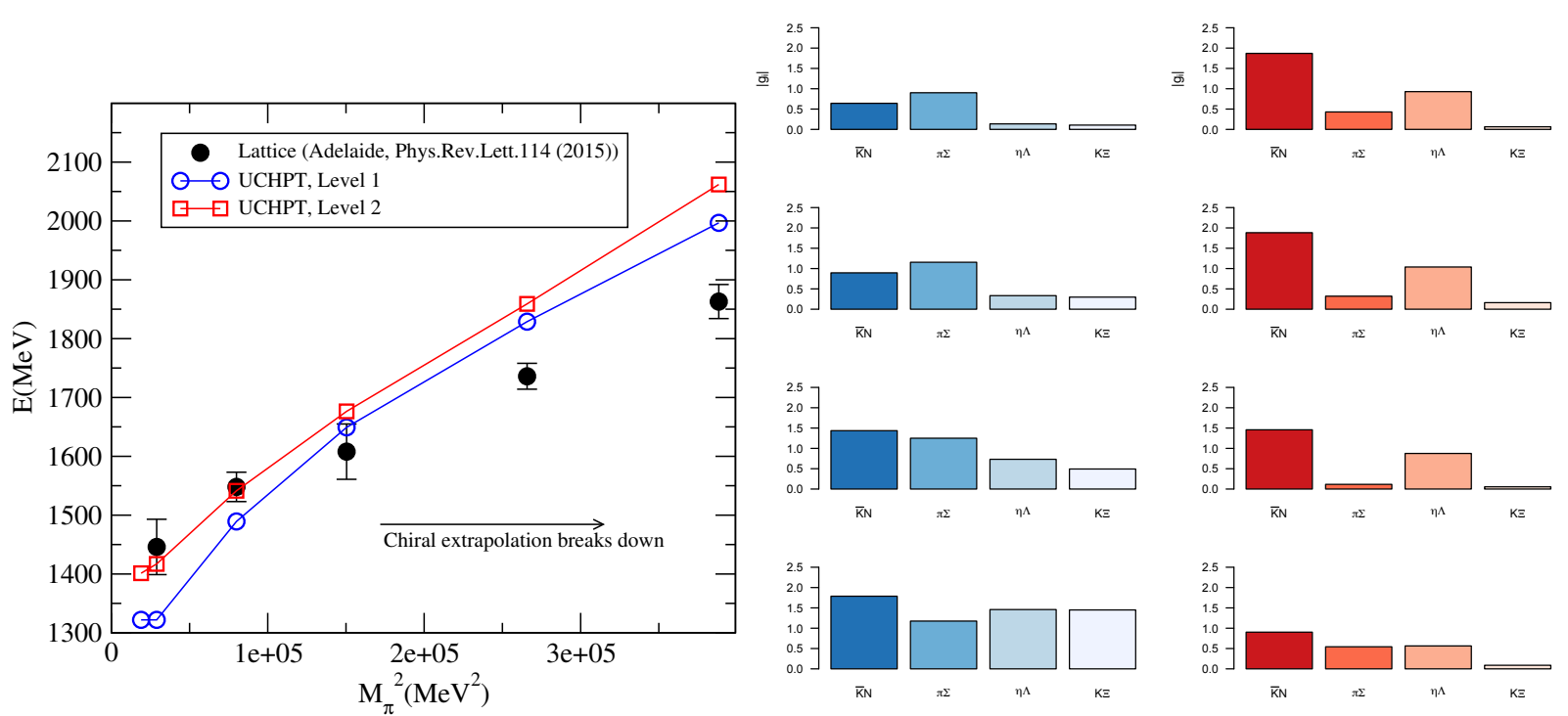

Figure 1: Left: Comparison between the U $\chi$ PT prediction and the Lattice data of Ref. [39] done for sets 1 to 5, and the physical set. Right: Couplings $\left|g_{i}\right|$ to the different channels of the first two energy levels found in the box for the pion masses from 170 to $515 \mathrm{MeV}$ (sets 1 to 4)

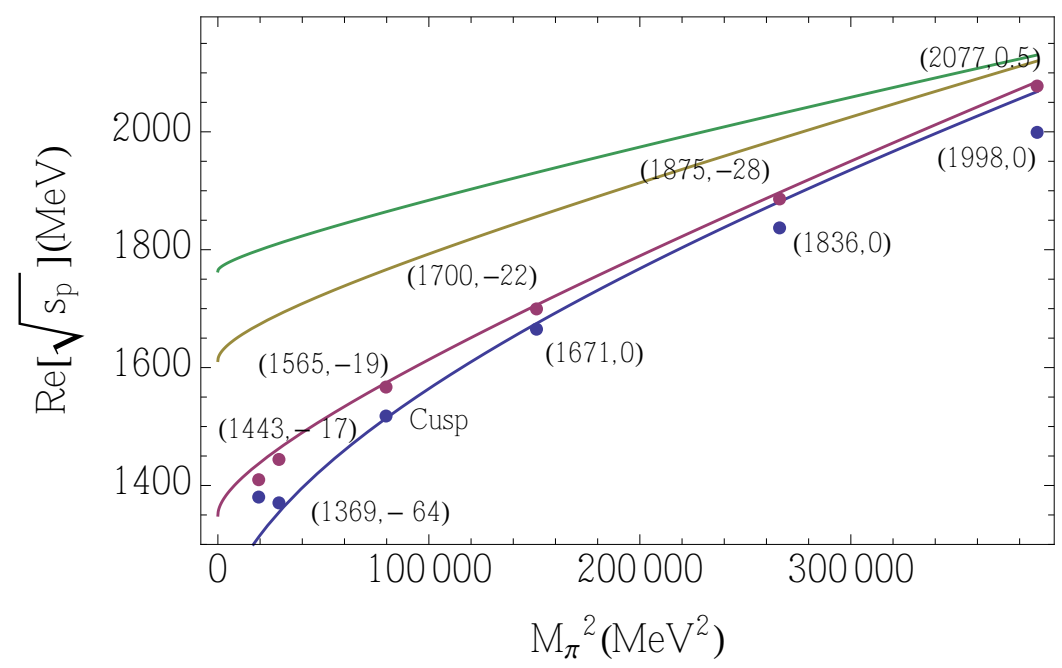

Figure 2: Behaviour of the the $\operatorname{Re} \sqrt{s}_{p}$ of the poles found in the $T$-matrix in the infinite volume with the $M_{\pi}^{2}$, for the physical, and 1 to 5 sets. The number between parenthesis indicates the pole position.

$\Lambda(1405)$. In any case, the existing data of Ref. [39] are predicted well and are compatible with the two-pole hypothesis for the $\Lambda(1405)$.

\section{References}

[1] R. H. Dalitz and S. F. Tuan, Annals Phys. 10, 307 (1960).

[2] R. H. Dalitz, T. C. Wong and G. Rajasekaran, Phys. Rev. 153, 1617 (1967).

[3] E. A. Veit, B. K. Jennings, A. W. Thomas and R. C. Barrett, Phys. Rev. D 31, 1033 (1985). 
Infinite volume

\begin{tabular}{|c|c|c|c|c|c|c|c|}
\hline \multirow[t]{2}{*}{ Set } & \multicolumn{7}{|c|}{ Channel } \\
\hline & Pole & $\bar{K} N$ & $\pi \Sigma$ & $\eta \Lambda$ & $K \Xi$ & $b_{\bar{K} N}$ & $b_{\pi \Sigma}$ \\
\hline \multirow[t]{2}{*}{$\mathrm{Ph}$. } & 1379-i 71 & 2.20 & 3.1 & 0.8 & 0.5 & 56 & -48 \\
\hline & 1412-i 19 & 3.1 & 1.7 & 1.5 & 0.3 & 23 & -81 \\
\hline \multirow[t]{2}{*}{1} & 1369-i 64 & 1.9 & 2.9 & 0.6 & 0.5 & 89 & -17 \\
\hline & 1443-i 17 & 2.6 & 1.35 & 1.32 & 0.3 & 15 & -91 \\
\hline \multirow[t]{2}{*}{2} & \multicolumn{5}{|c|}{ Cusp at 1518.34} & 64 & 0 \\
\hline & 1565-i 19 & 2.5 & 1.5 & 1.4 & 0.5 & 17 & -47 \\
\hline \multirow[t]{2}{*}{3} & 1671 & 2.0 & 1.3 & 1.1 & 0.6 & 39 & 9 \\
\hline & $1700-\mathrm{i} 22$ & 0.6 & 0.4 & 1.2 & 2.9 & 10 & -20 \\
\hline \multirow[t]{2}{*}{4} & 1836 & 1.9 & 1.2 & 1.7 & 1.8 & 48 & 33 \\
\hline & $1875-\mathrm{i} 28$ & 1.3 & 1.8 & 1.6 & 1.7 & 9 & -6 \\
\hline \multirow[t]{2}{*}{5} & 1998 & 0.9 & 0.8 & 1.9 & 2.9 & 92 & 75 \\
\hline & 2077-i 0.5 & 2.1 & 0.4 & 0.3 & 1.1 & 13 & -4 \\
\hline
\end{tabular}

Finite volume

\begin{tabular}{rrrrrrr}
\hline \hline \multicolumn{7}{c}{ Channel } \\
Pole & $\bar{K} N$ & $\pi \Sigma$ & $\eta \Lambda$ & $K \Xi$ & $b_{\bar{K} N}$ & $b_{\pi \Sigma}$ \\
1322 & 0.5 & 0.6 & 0.1 & 0.07 & 113 & 9 \\
1401 & 2.2 & 1.0 & 1.0 & 0.2 & 34 & -70 \\
\hline 1322 & 0.6 & 0.9 & 0.1 & 0.1 & 136 & 30 \\
1417 & 1.9 & 0.4 & 0.9 & 0.06 & 41 & -65 \\
\hline 1489 & 0.9 & 1.2 & 0.3 & 0.3 & 93 & 29 \\
1541 & 1.9 & 0.3 & 1.0 & 0.2 & 41 & -23 \\
\hline 1649 & 1.4 & 1.3 & 0.7 & 0.5 & 61 & 31 \\
1676 & 1.5 & 0.1 & 0.9 & 0.06 & 34 & 4 \\
\hline 1829 & 1.8 & 1.2 & 1.5 & 1.5 & 55 & 40 \\
1859 & 0.9 & 0.5 & 0.6 & 0.09 & 25 & 10 \\
\hline 1997 & 1.0 & 0.9 & 1.9 & 2.9 & 93 & 76 \\
2062 & 0.9 & 0.7 & 0.2 & 0.9 & 28 & 11 \\
\hline \hline
\end{tabular}

Table 3: Pole positions and couplings of the states $\left|g_{i}\right|$ in the infinite (left) and finite (right) volume for all sets.

[4] P. B. Siegel and B. Saghai, Phys. Rev. C 52, 392 (1995)

[5] K. Tanaka and A. Suzuki, Phys. Rev. C 45, 2068 (1992).

[6] Y. A. Chao, R. W. Kraemer, D. W. Thomas and B. R. Martin, Nucl. Phys. B 56, 46 (1973).

[7] R. H. Dalitz and J. G. McGinley, Proceedings of the international Conference on Hypernuclear and Kaon Physics (North Holland, Heidelberg, 1982)

[8] M. Bazzi, G. Beer, L. Bombelli, A. M. Bragadireanu, M. Cargnelli, G. Corradi, C. Curceanu (Petrascu) and A. d'Uffizi et al., Phys. Lett. B 704, 113 (2011)

[9] Y. Ikeda, T. Hyodo and W. Weise, Nucl. Phys. A 881, 98 (2012)

[10] M. Döring and U.-G. Meißner, Phys. Lett. B 704, 663 (2011)

[11] M. Mai, V. Baru, E. Epelbaum and A. Rusetsky, Phys. Rev. D 91, no. 5, 054016 (2015)

[12] N. Kaiser, T. Waas and W. Weise, Nucl. Phys. A 612, 297 (1997)

[13] J. A. Oller and U. G. Meißner, Phys. Lett. B 500, 263 (2001)

[14] E. Oset, A. Ramos and C. Bennhold, Phys. Lett. B 527, 99 (2002)

[15] N. Kaiser, P. B. Siegel and W. Weise, Nucl. Phys. A594 (1995) 325.

[16] E. Oset and A. Ramos, Nucl. Phys. A 635, 99 (1998)

[17] D. Jido, A. Hosaka, J. C. Nacher, E. Oset and A. Ramos, Phys. Rev. C 66, 025203 (2002)

[18] D. Jido, J. A. Oller, E. Oset, A. Ramos and U.-G. Meiß ner, Nucl. Phys. A 725, 181 (2003).

[19] C. Garcia-Recio, J. Nieves, E. Ruiz Arriola and M. J. Vicente Vacas, Phys. Rev. D 67, 076009 (2003) 
[20] M. Mai and U. G. Meißner, Nucl. Phys. A 900, 51 (2013)

[21] P. J. Fink, Jr., G. He, R. H. Landau and J. W. Schnick, Phys. Rev. C 41, 2720 (1990).

[22] M. Döring, D. Jido and E. Oset, Eur. Phys. J. A 45, 319 (2010)

[23] B. Borasoy, U.-G. Meißner and R. Nißler, Phys. Rev. C 74, 055201 (2006)

[24] L. S. Geng and E. Oset, Eur. Phys. J. A 34, 405 (2007)

[25] V. K. Magas, E. Oset and A. Ramos, Phys. Rev. Lett. 95, 052301 (2005).

[26] S. Prakhov et al. [Crystall Ball Collaboration], Phys. Rev. C 70, 034605 (2004).

[27] T. Hyodo, A. Hosaka, E. Oset, A. Ramos and M. J. Vicente Vacas, Phys. Rev. C 68, 065203 (2003)

[28] T. Hyodo, A. Hosaka, M. J. Vicente Vacas and E. Oset, Phys. Lett. B 593, 75 (2004).

[29] D. Jido, E. Oset and T. Sekihara, Eur. Phys. J. A 42, 257 (2009).

[30] T. Sekihara, T. Hyodo and D. Jido, PTEP 2015, 063 D04 (2015)

[31] T. Hyodo, D. Jido and A. Hosaka, Phys. Rev. C 78 (2008) 025203.

[32] J. Bulava, R. G. Edwards, E. Engelson, B. Joo, H. W. Lin, C. Morningstar, D. G. Richards and S. J. Wallace, Phys. Rev. D 82, 014507 (2010)

[33] B. J. Menadue, W. Kamleh, D. B. Leinweber and M. S. Mahbub, Phys. Rev. Lett. 108, 112001 (2012)

[34] G. P. Engel et al. [BGR (Bern-Graz-Regensburg) Collaboration], Phys. Rev. D 87, no. 3, 034502 (2013)

[35] G. P. Engel et al. [BGR Collaboration], Phys. Rev. D 87, no. 7, 074504 (2013)

[36] R. G. Edwards et al. [Hadron Spectrum Collaboration], Phys. Rev. D 87, no. 5, 054506 (2013)

[37] W. Melnitchouk, S. O. Bilson-Thompson, F. D. R. Bonnet, J. N. Hedditch, F. X. Lee, D. B. Leinweber, A. G. Williams and J. M. Zanotti et al., Phys. Rev. D 67, 114506 (2003)

[38] A. Walker-Loud, H.-W. Lin, D. G. Richards, R. G. Edwards, M. Engelhardt, G. T. Fleming, P. Hagler and B. Musch et al., Phys. Rev. D 79, 054502 (2009)

[39] J. M. M. Hall, W. Kamleh, D. B. Leinweber, B. J. Menadue, B. J. Owen, A. W. Thomas and R. D. Young, Phys. Rev. Lett. 114, no. 13, 132002 (2015)

[40] M. Döring, J. Haidenbauer, U.-G. Meißner and A. Rusetsky, Eur. Phys. J. A 47, 163 (2011)

[41] M. Lage, U. G. Meißner and A. Rusetsky, Phys. Lett. B 681, 439 (2009)

[42] D. N. Tovee et al., Nucl. Phys. B33 (1971) 493.

[43] R. J. Nowak et al., Nucl. Phys. B139 (1978) 61.

[44] J. Nebreda and J. R. Pelaez., Phys. Rev. D 81, 054035 (2010)

[45] M. Doring, U. -G. Meissner, E. Oset and A. Rusetsky, Eur. Phys. J. A 47, 139 (2011).

[46] A. Martinez Torres, L. R. Dai, C. Koren, D. Jido and E. Oset, Phys. Rev. D 85, 014027 (2012)

[47] J. A. Oller, E. Oset and J. R. Pelaez, Phys. Rev. D 59, 074001 (1999) 